\title{
Self-Assembled, Dilution-Responsive Hydrogels for Enhanced Thermal Stability of Insulin Biopharmaceuticals
}

\author{
Catherine M. Meis, Erika E. Salzman, Caitlin L. Maikawa, Anton A. A. Smith, Joseph L. Mann,
} Abigail K. Grosskopf, and Eric A. Appel*

Cite This: ACS Biomater. Sci. Eng. 2021, 7, 4221-4229

Read Online

ABSTRACT: Biotherapeutics currently dominate the landscape of new drugs because of their exceptional potency and selectivity. Yet, the intricate molecular structures that give rise to these beneficial qualities also render them unstable in formulation. Hydrogels have shown potential as stabilizing excipients for biotherapeutic drugs, providing protection against harsh thermal conditions experienced during distribution and storage. In this work, we report the utilization of a cellulose-based supramolecular hydrogel formed from polymernanoparticle (PNP) interactions to encapsulate and stabilize insulin, an important biotherapeutic used widely to treat diabetes.
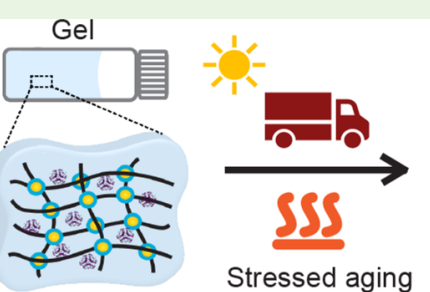

$\mathfrak{3}_{3}=$ Insulin

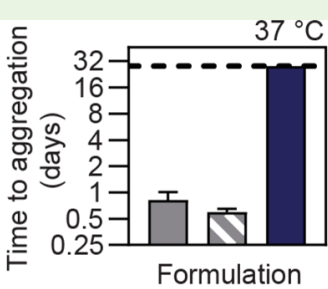

Insulin $(10 \mathrm{mg} / \mathrm{ml})$

Humulin $(3.47 \mathrm{mg} / \mathrm{ml})$ Insulin w/ hydrogel Encapsulation of insulin in these hydrogels prevents insulin aggregation and maintains insulin bioactivity through stressed aging conditions of elevated temperature and continuous agitation for over 28 days. Further, insulin can be easily recovered by dilution of these hydrogels for administration at the point of care. This supramolecular hydrogel system shows promise as a stabilizing excipient to reduce the cold chain dependence of insulin and other biotherapeutics.

KEYWORDS: supramolecular, drug delivery, biotherapeutic, cold chain, biomaterials, hydrogel

\section{INTRODUCTION}

Biotherapeutics have grown to be a dominant portion of the pharmaceutical market over the past two decades. In 2018, eight of the top ten grossing drug products globally were biotherapeutics. While the complex and intricate structures of these macromolecular drugs result in pharmaceutical products with exceptional potency and selectivity, it also makes them inherently susceptible to loss of activity due to thermal denaturation and aggregation., ${ }^{2,3}$ Exposure to high, or even ambient, temperatures during transportation, storage, and use can cause partial or complete loss of bioactivity. To prevent loss of activity, most biotherapeutics require refrigerated shipping and storage throughout the supply chain, referred to as the "cold chain", which reduces accessibility to regions of the world with limited infrastructure. ${ }^{4-7}$ Additionally, cold chain logistics are costly, with an estimated $\$ 15.7$ billion spent globally by biopharmaceutical companies on cold chain logistics alone in 2019. ${ }^{8}$ Improved thermal stability of biotherapeutics would enable increased global access to critical drugs and reduce storage and transportation costs.

There are many approaches to improving the stability of biotherapeutic formulations, but typical strategies include modifying the drug molecule itself, altering its physical state, or formulating with excipients-often in combination-such as salts, amino acids, polymers, and surfactants. ${ }^{9,10}$ One prevalent method is to use protein engineering to design biotherapeutic analogues. ${ }^{10}$ While this has been successful, it carries the risk of altered drug potency or immunogenicity. ${ }^{3}$ Another commonly used stabilization technique is lyophilization, or freeze-drying, of the drug into a dry powder prior to shipping and long-term storage. ${ }^{1,12}$ Unfortunately, lyophilization is not ideal for many drug products because the drastic changes in temperature, pressure, and hydration state during processing can result in activity loss. Liquid formulations of biotherapeutics incorporate stabilizing excipients; $;^{9,11-13}$ however, many currently used pharmaceutical excipients were developed for use with small molecule drugs and were not optimized to stabilize proteins. Therefore, there is a need to develop next-generation excipients specifically designed to enhance the stability of biotherapeutics, especially for parenteral formulations. The drug delivery field has made considerable advances in the formulation of biotherapeutics in soft materials to modulate drug pharmacokinetics; this body of knowledge and materials could be leveraged to advance materials for biostability as well. .,3,14-19 $^{-19}$

Special Issue: Advanced Biomedical Hydrogels

Received: September 3, 2020

Accepted: November 4, 2020

Published: November 17, 2020

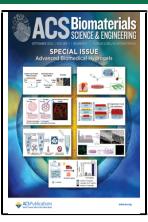



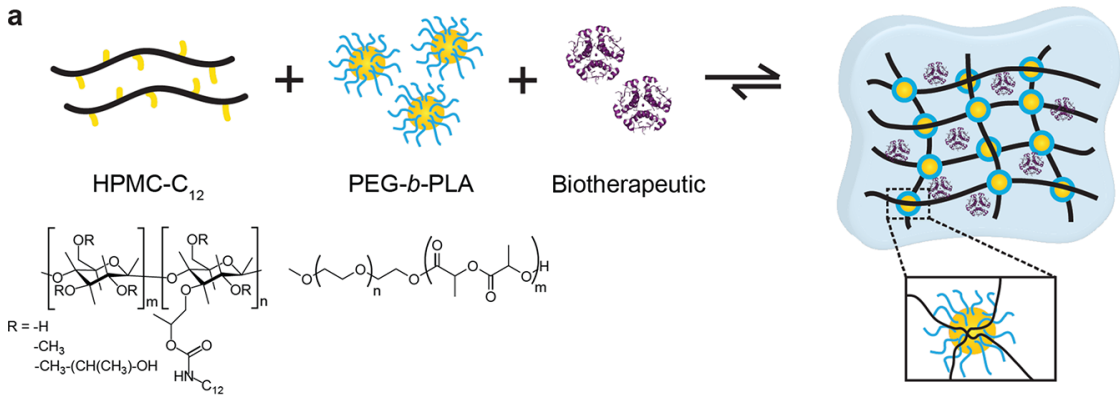

b
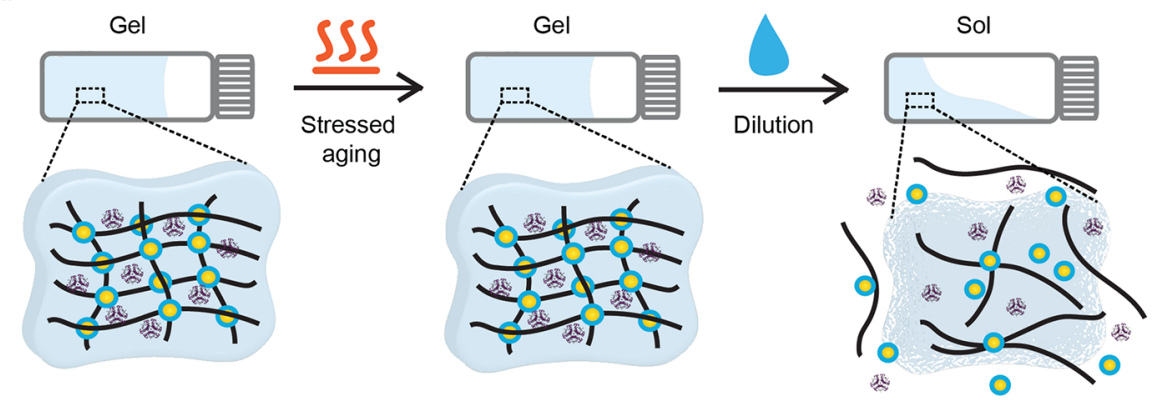

Figure 1. (a) Supramolecular polymer-nanoparticle (PNP) hydrogels composed of (hydroxypropyl)methyl cellulose (HPMC) with dodecyl side chain modifications as the network polymer that is physically cross-linked by bridging interactions with poly(ethylene glycol)-block-poly(lactic acid) (PEG-b-PLA) diblock copolymer nanoparticles. Biotherapeutics, such as insulin (shown), can be encapsulated in these hydrogels during precursor mixing. (b) Schematic indicating the desired performance of our thermally stabilizing supramolecular hydrogel excipient.

Recently, hydrogels have been utilized to improve the thermal stability of biotherapeutics. Nanogels, nanoparticles, microparticles, and hydrogel films are forms of hydrogel excipients used to stabilize the protein of interest and can reduce cold chain dependence. ${ }^{20-25}$ Hydrogels are promising candidates for biotherapeutic stabilization because they can maintain the proteins in their native aqueous environment while promoting stabilizing interactions between the hydrogel polymer backbone and protein. A poly(ethylene glycol) (PEG)-based covalent hydrogel network with a photolabile moiety has been reported to stabilize biotherapeutic cargo at elevated temperatures. ${ }^{26}$ Upon exposure to ultraviolet light, the hydrogel network backbone degraded to afford biotherapeutic recovery. While the system effectively stabilized the selected enzymes, the presence of degraded hydrogel material may prove to be an obstacle for clinical translation as the in vivo biocompatibility has not been assessed. Both trehalose-based hydrogels and hydrogels with trehalose side chain modifications have also shown utility as thermal stabilizing excipients for a variety of proteins, including insulin. ${ }^{27-29}$ Unfortunately, these systems have not been shown to be injectable or degradable in vivo. To use hydrogels as stabilizing excipients in parenteral biotherapeutic formulations, there is still a need to design materials that allow mild encapsulation, are injectable, are biocompatible in vivo, and easily release the drug without unintentionally altering drug pharmacokinetics.

We have developed a supramolecular hydrogel platform, based upon polymer-nanoparticle (PNP) interactions between hydrophobically modified cellulose-derived polymers and core-shell polymeric nanoparticles, as an encapsulating excipient (Figure 1a). ${ }^{30-33}$ These supramolecular hydrogels have been used for a variety of biomedical applications and are biocompatible, injectable, and readily tunable in mechanical properties as characterized by rheology. ${ }^{34-37}$ We can readily encapsulate biotherapeutic cargo into these hydrogels under mild conditions by simply mixing the drug into the bulk material. Further, the hydrogel can be diluted to disrupt the network and yield a drug-containing solution for administration, as the various hydrogel components are Generally Recognized as Safe (GRAS-listed) by the FDA and are nontoxic. These qualities indicate that our PNP hydrogel network is a promising candidate for a translatable stabilizing excipient for biotherapeutics.

In this study, we demonstrate that PNP hydrogels can stabilize insulin against thermal aggregation and denaturation. Insulin is a clinically relevant biotherapeutic that is required by over 150 million patients living with diabetes worldwide, but suffers from thermal instability and is highly prone to aggregate into insoluble, inactive and immunogenic amyloid fibrils when it is exposed to increased temperatures or agitation. ${ }^{13,38-41}$ Stabilized insulin capable of maintaining drug integrity in environmental conditions could increase access to this drug in regions of the world such as Africa and the Western Pacific where diabetes prevalence is increasing but reliable cold chain storage and transportation is challenging. ${ }^{42}$ We investigate the thermal stability of insulin encapsulated in these hydrogels with stressed aging experiments under relevant simulated environmental conditions to demonstrate the possibility for cold chain independence (Figure 1b). We show that these supramolecular hydrogels are promising for use as thermal stabilization agents in insulin formulations, and potentially other parenteral biotherapeutic formulations as well.

\section{MATERIALS AND METHODS}

Materials. Racemic lactide (3,6-dimethyl-1,4-dioxane-2,5-dione, $99 \%$, Sigma-Aldrich) was recrystallized twice from dry ethyl acetate to remove impurities, with sodium sulfate included as a desiccant prior to the first recrystallization. Dichloromethane (DCM) was dried immediately before use by cryogenic distillation. Recombinant human insulin was purchased from Gibco as a lyophilized powder containing zinc and then reconstituted. Humulin $\mathrm{R}(100 \mathrm{U} / \mathrm{ml}, 3.47 \mathrm{mg} / \mathrm{mL}$, Eli 
Lilly) was used as received. All other materials were purchased from Sigma-Aldrich and used as received.

Synthesis of PEG-b-PLA block copolymers. Poly(ethylene glycol)-block-poly(lactic acid) (PEG-b-PLA) block copolymers were synthesized by ring-opening polymerization of lactide onto a PEG macromer, as described previously. ${ }^{30}$ Dry PEG methyl ether (avg. $M_{\mathrm{n}}$ $=5000 \mathrm{~g} / \mathrm{mol}, 0.5 \mathrm{~g}, 0.1 \mathrm{mmol})$ and 1,8-diazabicyclo[5.4.0] undec-7ene (DBU, 98\%, $15 \mu \mathrm{L}, 0.1 \mathrm{mmol}$ ) were dissolved together in dry DCM $(1.5 \mathrm{~mL})$; twice recrystallized racemic lactide $(2 \mathrm{~g}, 13.9 \mathrm{mmol})$ was dissolved separately in dry DCM $(9 \mathrm{~mL})$. The PEG/DBU solution was quickly added to the lactide solution under nitrogen atmosphere and stirred rapidly for $8 \mathrm{~min}$ then quenched with dilute acetic acid in acetone $(100 \mu \mathrm{L})$. The polymer was recovered by precipitating twice from a 50:50 diethyl ether: hexanes solution and dried under vacuum. Target molecular weight of the block copolymers by DMF SEC: $22.5-27.5 \mathrm{kDa}$ (5 kDa PEG, 17.5-22.5 kDa PLA with $Đ<1.2$ ) (Figures $S 1-S 4$ ).

DMF-SEC Measurements. $M_{\mathrm{n}}$ and $M_{\mathrm{w}}$ were determined after passing through two size exclusion chromatography columns (Resolve Mixed Bed Low DVB, ID $7.8 \mathrm{~mm}, M_{\mathrm{w}}$ range $200-600000 \mathrm{~g} \mathrm{~mol}^{-1}$ (Jordi Laboratories)) in a mobile phase of $N, N$-dimethylformamide (DMF) with $0.1 \mathrm{M} \mathrm{LiBr}$ at $35{ }^{\circ} \mathrm{C}$ and a flow rate of $1.0 \mathrm{~mL} \mathrm{~min}{ }^{-1}$ (Dionex Ultimate 3000 pump, degasser, and autosampler (Thermo Fisher Scientific)) using linear polyethylene glycol standards (American Polymer Standards Corporation).

Synthesis of Dodecyl-Modified HPMC (HPMC- $\mathrm{C}_{12}$ ). Dodecylmodified (hydroxypropyl)methyl cellulose (HPMC) was synthesized as previously described. ${ }^{30}$ Briefly, HPMC (hypromellose, USP grade, $1 \mathrm{~g}$ ) was dissolved in 1-methyl-2-pyrrolodinone (NMP, $40 \mathrm{~mL}$ ) at 90 ${ }^{\circ} \mathrm{C}$. Dodecyl isocyanate $(99 \%, 125 \mu \mathrm{L}, 0.52 \mathrm{mmol})$ dissolved in NMP $(5 \mathrm{~mL}$ ) was added dropwise to the heated reaction vessel followed by 3 drops of $N, N$-diisopropyethylamine ( $\geq 99 \%$, Hünig's base) catalyst. Reaction solution was allowed to cool to room temperature and stirred overnight. HPMC- $\mathrm{C}_{12}$ was recovered by precipitation in acetone and purified by dialysis (MWCO $3500 \mathrm{kDa}$ ) for 4 days (Figure S3). Lyophilized HPMC- $\mathrm{C}_{12}$ was redissolved in phosphate buffered saline (PBS) as a $6 \mathrm{wt} \%$ stock solution. Representative ${ }^{1} \mathrm{H}$ NMR analysis has been previously published. ${ }^{3}$

PEG-b-PLA Nanoparticle (NP) Synthesis by Nanoprecipitation. To form core-shell NPs, PEG-PLA (50 mg) was dissolved in a 75:25 acetonitrile:DMSO solution $(1 \mathrm{~mL})$ and added dropwise to ultrapure water $(10 \mathrm{~mL})$ at a high stir rate $(600 \mathrm{rpm})$. The NP solution was centrifuged over a filter (Amicon Ultra-15, threshold molecular weight $10 \mathrm{kDa}$ ) and resuspended in PBS as a $20 \mathrm{wt} \%$ stock solution (total volume $250 \mu \mathrm{L}$ per batch). The resulting NPs were characterized by dynamic light scattering (DLS, DynaPro II plate reader, Wyatt Technology) (hydrodynamic diameter $=30-60 \mathrm{~nm}$; PDI $<0.2$ ).

Supramolecular PNP Hydrogel Formulation and Drug Loading. Supramolecular PNP hydrogels were formulated by combining the HPMC- $\mathrm{C}_{12}$ stock solution and PEG-PLA NP stock solution diluted to the desired concentration via elbow mixing as previously described. ${ }^{34}$ PNP hydrogel formulations are designated by HPMC-C $_{12}$ wt \%: NP wt \%. For example, a 2:10 PNP hydrogel is $2 \mathrm{wt}$ $\%$ HPMC-C $\mathrm{C}_{12}$ and $10 \mathrm{wt} \% \mathrm{NPs}$ ( $12 \mathrm{wt} \%$ total solid polymer) of the total formulation mass, with the remainder comprised of PBS or drug cargo solution. HPMC- $\mathrm{C}_{12}$ stock solution $(400 \mathrm{mg})$ was loaded into a $3 \mathrm{~mL}$ luer lock syringe. NP stock solution $(600 \mu \mathrm{L})$ was combined with PBS $(200 \mu \mathrm{L})$ and loaded into a $3 \mathrm{~mL}$ luer lock syringe. The HPMC- $\mathrm{C}_{12}$-filled syringe was connected to a female luer $\times$ female luer elbow fitting, and the solution was pushed through the elbow to eliminate air in the connection before attaching the NP-filled syringe to the other side. The solutions were mixed through the elbow at a relatively fast rate for $>50$ cycles $(1$ cycle $=$ complete transfer of material from one syringe to the other and then back into original syringe) until a homogeneous hydrogel was formed. All other hydrogel formulations were prepared identically at the appropriate concentrations. For insulin-loaded hydrogels, reconstituted insulin and the molecular probe thioflavin $\mathrm{T}$ ( $\mathrm{ThT}$, dye content $\geq 65 \%$ ) were mixed into the hydrogel formulation by addition to the NP-filled syringe. Insulin stock solution $(40 \mathrm{mg} / \mathrm{mL})$ was prepared from lyophilized powder by first dissolving in $0.12 \mathrm{M}$ hydrochloric acid and then neutralizing with $0.13 \mathrm{M}$ sodium hydroxide. Concentrated phosphate buffer $(0.45 \mathrm{M})$, concentrated sodium chloride $(135 \mathrm{mg}$ / $\mathrm{mL}$ ), and ultrapure water were added to achieve final concentrations matching PBS (10 $\mathrm{mM}$ phosphate, $0.9 \% \mathrm{NaCl}$ ). Neutral $\mathrm{pH}$ was verified using $\mathrm{pH}$ strips. Insulin was loaded into the hydrogels to a final concentration of $10 \mathrm{mg} / \mathrm{mL}$, except 2:10 hydrogels were loaded at $6.7 \mathrm{mg} / \mathrm{mL}$. ThT $(640 \mu \mathrm{g} / \mathrm{mL}, 2 \mathrm{mM}$ stock solution in PBS) was added to a final concentration of $25 \mu \mathrm{M}$ for all samples $(15 \mu \mathrm{L}$ per 1.2 $\mathrm{mL}$ sample volume).

Rheological Characterization of PNP Hydrogels. Rheological measurements were performed on a TA Instruments Discovery Hybrid Rheometer (DHR-2) with a $40 \mathrm{~mm} 2^{\circ}$ cone geometry with a fixed gap of $51 \mu \mathrm{m}$. Amplitude sweeps were measured at a fixed frequency of $1 \mathrm{~s}^{-1}$ (Figure S5). Flow sweeps were measured at shear rates from $100-0.01 \mathrm{~s}^{-1}$. Frequency sweeps were measured at a constant torque of $5 \mu \mathrm{N} \mathrm{m}$ from $0.0628-628 \mathrm{rad} \mathrm{s}^{-1}$. To measure the changes in hydrogel rheological properties upon dilution, the hydrogel sample was recovered from the rheometer and serially diluted with PBS using the elbow mixing method described for preparing the supramolecular PNP hydrogel formulations.

In Vitro Insulin Fibrillation Assay. Insulin fibrillation was characterized in vitro by thioflavin $\mathrm{T}$ (ThT) assay. In the presence of insulin amyloid fibrils, ThT produces a strong fluorescence signal, yielding a binary assay result indicating the presence or absence of fibrils (Figure S6). ${ }^{43}$ Insulin-loaded hydrogels and assay controls, all preformulated with ThT, were loaded into 96 -well black plates (200 $\mu \mathrm{L}$ per well, $n=4$ ), sealed, and subjected to stressed aging conditions. To simulate hot ambient conditions, plated samples were subjected to continuous linear shaking $(410 \mathrm{cpm}, 5 \mathrm{~mm})$ at $37{ }^{\circ} \mathrm{C}$ in a BioTek Synergy H1 Hybrid Multi-Mode Plate Reader. Data was acquired at $20 \mathrm{~min}$ intervals by excitation at $450 \mathrm{~nm}$ and collection of emitted signal at $482 \mathrm{~nm}$. To simulate harsh environmental conditions, plated samples were subjected to $50{ }^{\circ} \mathrm{C}$ and continuous agitation at $150 \mathrm{rpm}$ on a shaker plate inside a humidified incubator. Fluorescence readings were acquired daily ( read at $10 \mathrm{~min}$ intervals for $1 \mathrm{~h}$ at $45^{\circ} \mathrm{C}$ ) for the first week, and every 2-3 days until the end point of the study. Aging studies were terminated when the samples appeared to be significantly dehydrated by visual inspection. Trehalose, a common excipient, was tested at 2, 6, and $12 \mathrm{wt} \%$ trehalose (chosen to match total polymer solid content in certain hydrogel formulations) with $10 \mathrm{mg} / \mathrm{mL}$ insulin at $37{ }^{\circ} \mathrm{C}$. Humulin $(3.47 \mathrm{mg} / \mathrm{mL})$, a standard low-cost commercially available formulation, was tested at $37^{\circ} \mathrm{C}$. Stability data is shown as mean fluorescence signal over time $(n=4)$. Time to aggregation was determined by defining an arbitrary signal threshold (750 000 arbitrary fluorescence units, AFU).

Circular Dichroism. Circular dichroism was used to confirm that gel-encapsulated insulin retained its secondary protein structure postaging. Selected insulin-loaded hydrogel samples from the in vitro insulin fibrillation plate assay conducted at $37^{\circ} \mathrm{C}$ were diluted to $0.2-$ $0.5 \mathrm{mg} / \mathrm{mL}$ insulin in PBS for analysis and compared to a freshly prepared recombinant insulin standard. Near-UV circular dichroism spectroscopy (J-815 CD Spectropolarimeter, Jasco Corporation) was performed at $20{ }^{\circ} \mathrm{C}$ over $190-260 \mathrm{~nm}$ wavelengths using a $0.1 \mathrm{~cm}$ path-length cell.

In Vitro Insulin Cellular Activity Assay. C2C12 mouse muscle myoblasts (ATCC CRL-1772) were cultured to confirm insulin functional activity post-aging via the AKT (protein kinase B) phosphorylation pathway using AlphaLISA SureFire Ultra (PerkinElmer) kits for detection of phosphorylated AKT 1/2/3 (pS473) compared to total AKT, based upon methods described previously. ${ }^{39,40}$ Cells were confirmed to be free of mycoplasma contamination prior to use. Dulbecco's Modified Eagle's Medium (DMEM) (4.5 g/L D-glucose, L-glutamine, and $110 \mathrm{mg} / \mathrm{L}$ sodium pyruvate, Gibco) was supplemented with $10 \%$ fetal bovine serum (FBS) and 5\% penicillin-streptomycin to formulate complete culture media. Cells were seeded at a density of 25000 cells/well in a volume of $200 \mu \mathrm{L} /$ well in a 96-well tissue culture plate and grown for $24 \mathrm{~h}$. Prior to insulin stimulation, the cells were washed twice with $200 \mu \mathrm{L}$ 
a
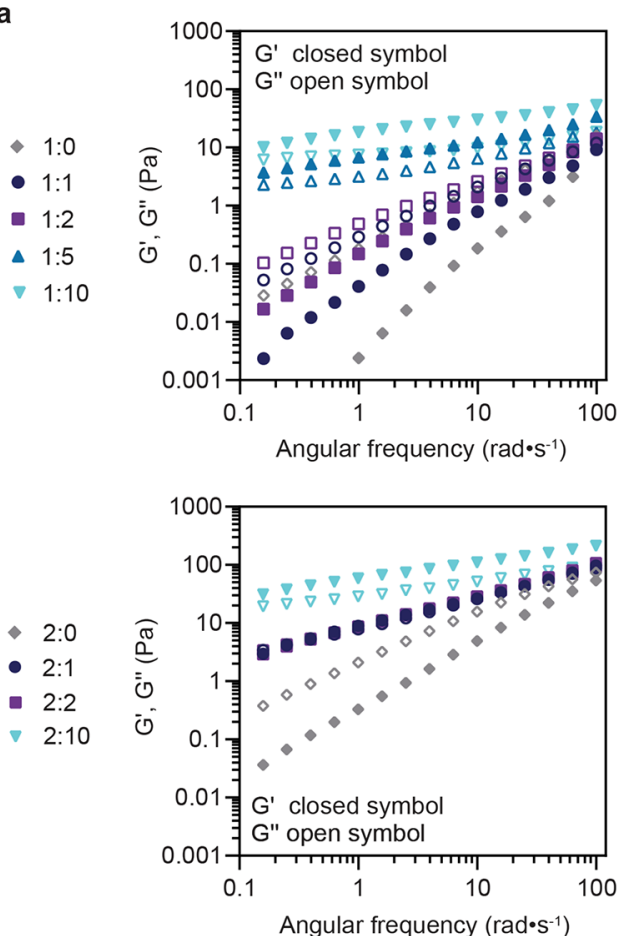

b
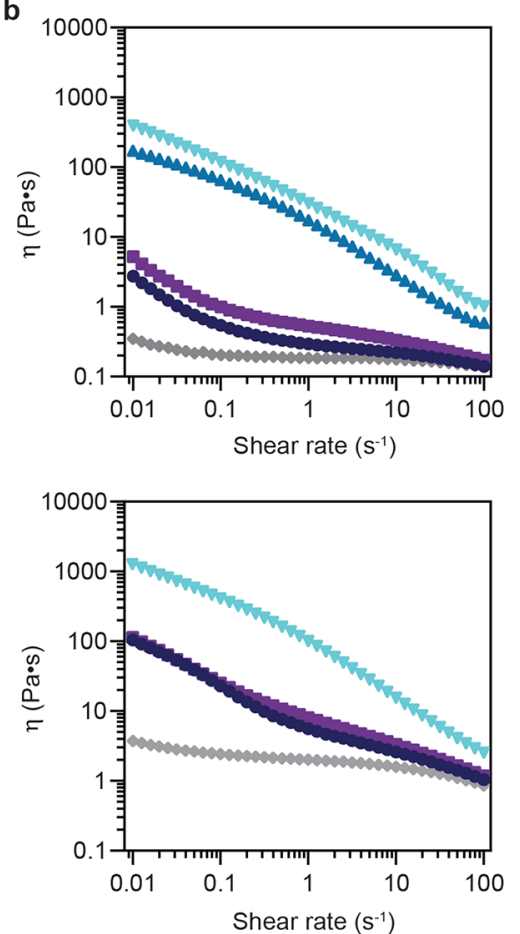

Figure 2. Rheological characterization of 1 wt \% polymer and 2 wt \% polymer PNP hydrogel formulations with varying wt \% NPs. Hydrogel formulations are denoted as P:NP, where $\mathrm{P}=$ wt \% polymer and $\mathrm{NP}=\mathrm{wt} \%$ nanoparticles. Neat polymer solutions are denoted as 1:0 and 2:0 formulations. (a) Frequency-dependent oscillatory shear measurements indicate the hydrogel storage $\left(G^{\prime}\right)$ and loss $\left(G^{\prime \prime}\right)$ moduli increase with increasing wt \% NPs. (b) Steady-shear measurements of all hydrogel formulations exhibit significant shear-thinning behavior that collapses to the shear viscosity values of the neat polymer solutions.

of unsupplemented DMEM and starved in $100 \mu \mathrm{L}$ of unsupplemented DMEM overnight. Insulin test samples were formulated by serial dilution in unsupplemented DMEM to the desired concentrations for the dose-response curve. The media was then removed and the cells were stimulated with $100 \mu \mathrm{L}$ of each insulin test sample for $30 \mathrm{~min}$ while incubating at $37^{\circ} \mathrm{C}$. Cells were washed twice with $100 \mu \mathrm{L}$ of cold $1 \times$ Tris-buffered saline before adding $100 \mu \mathrm{L}$ of lysis buffer to each well and shaking for at least $10 \mathrm{~min}$ at room temperature to fully lyse cells. Thirty microliters of lysate was transferred to a 96-well white half-area plate for each assay. Each sample was assayed to determine both [pAKT] and [total AKT1]. Assays were completed according to the manufacturer's protocol. Plates were incubated at room temperature and read $18-20 \mathrm{~h}$ after the addition of the final assay reagents using a Tecan Infinite M1000 PRO plate reader with standard AlphaLISA protocol. Data $(n=3$ aged sample replicates from $37^{\circ} \mathrm{C}$ stressed aging in vitro insulin fibrillation plate assay, $n=1$ cellular assay replicates) was plotted as the ratio of $[\mathrm{pAKT}] /[$ total AKT1] and normalized to a scale of 0 to $1 . \operatorname{logEC50}$ values were determined using the log[agonist] versus response variable slope (four parameters) regression in GraphPad Prism 8.0.2.

Statistical Analysis Methods. Statistical significance was assessed using the Extra sum-of-squares F-test to determine if the $\operatorname{logEC} 50$ differed between data sets. Data sets were compared in pairs and Bonferroni posthoc tests were used to adjust for multiple comparisons $($ alpha $=0.01)$.

\section{RESULTS AND DISCUSSION}

PNP Hydrogel Formulation Effects on Rheology. The ideal hydrogel drug product for insulin stabilization and delivery would be able to encapsulate the drug in a mild manner, inject easily for processability, maintain insulin stability in the vial, and readily disperse for facile subcutaneous delivery. In terms of rheological properties, the hydrogel should be shear-thinning and mechanically tunable. Polymer- nanoparticle (PNP) hydrogels are formed by bridging of hydrophobically modified (hydroxypropyl)methyl cellulose (HPMC) polymers between poly(ethylene glycol)-block-poly(lactic acid) (PEG-b-PLA) diblock copolymer NPs, whereby the HPMC polymers exhibit dynamic, multivalent interactions with the surface of the NPs (Figure 1a). These PNP hydrogels have previously been shown to exhibit both shear-thinning and self-healing properties that enable facile processing and administration by injection or spraying. . $^{19,30,34-36,44,45}$ In addition, the viscoelastic properties of these materials have been shown to be easily tunable by varying the PNP hydrogel formulation.

In the present work, a set of PNP hydrogels with distinct rheological properties were formulated by varying the total polymer and NP content, denoted as $\mathrm{P}: \mathrm{NP}$, where $\mathrm{P}=$ wt $\%$ polymer and $\mathrm{NP}=$ wt \% nanoparticles (neat polymer solutions denoted X:0). Viscoelastic properties (storage modulus, $G^{\prime}$, and loss modulus, $G^{\prime \prime}$ ) of the resulting hydrogels were characterized by frequency sweep within the linear viscoelastic regime and were consistent with those observed previously (Figures 2a, S5, and S6). In general, 2 wt \% polymer (2:X) hydrogel formulations exhibited higher moduli than 1 wt \% (1:X) formulations due to increased network polymer content. Increasing NP content from $1 \mathrm{wt} \%$ to $10 \mathrm{wt} \%$ resulted in an increase in both the storage and loss modulus values. 1:5, 1:10, and 2:10 PNP hydrogels exhibited solid-like behavior $\left(G^{\prime}>\right.$ $\left.G^{\prime \prime}\right)$ across the entire frequency range; $1: 1,1: 2,2: 1$, and $2: 2$ hydrogel formulations were non-gelling $\left(G^{\prime \prime}>G^{\prime}\right)$, though $G^{\prime}$ and $G^{\prime \prime}$ approach a crossover point near $100 \mathrm{rad} \mathrm{s}^{-1}$.

All PNP hydrogel formulations exhibited shear-thinning behavior with increasing shear rate for the range tested, with the viscosity collapsing onto that of the neat polymer solution 
a
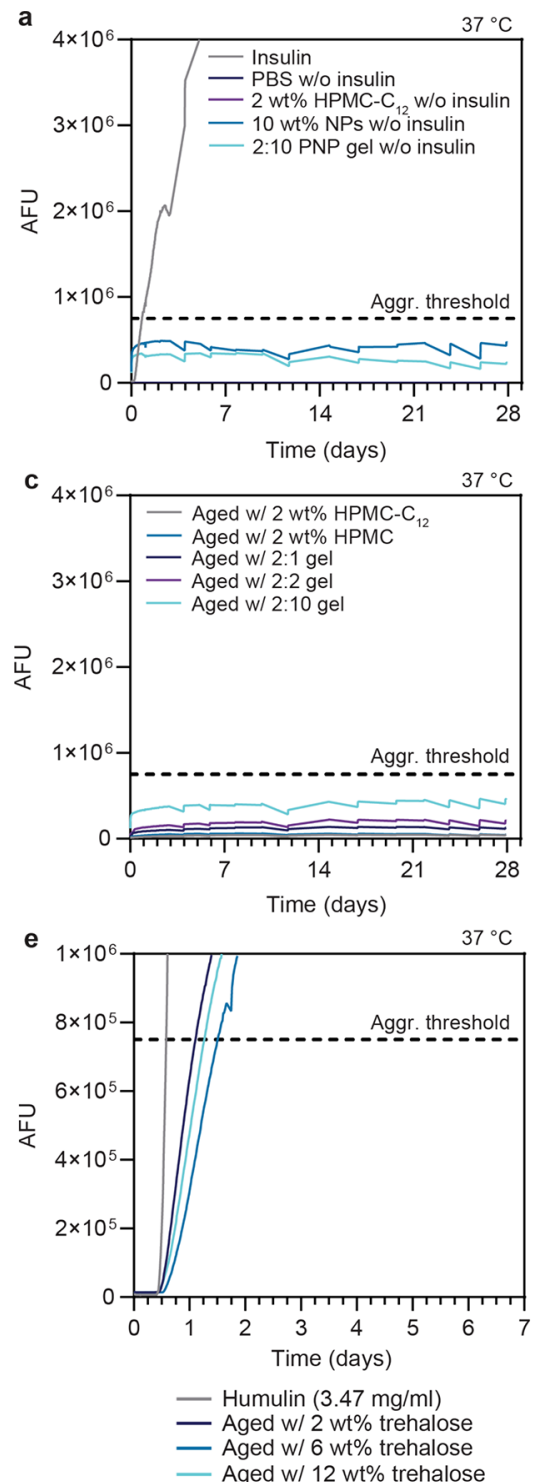

b
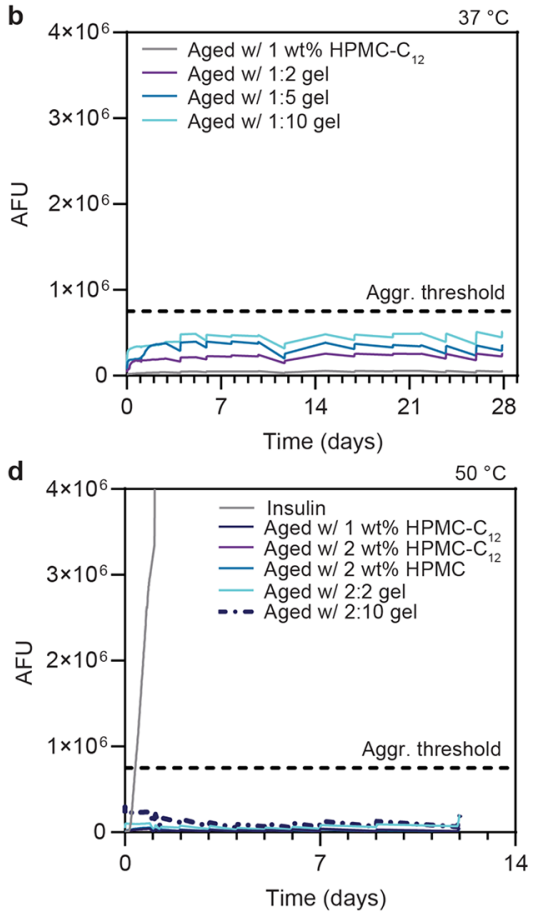

f

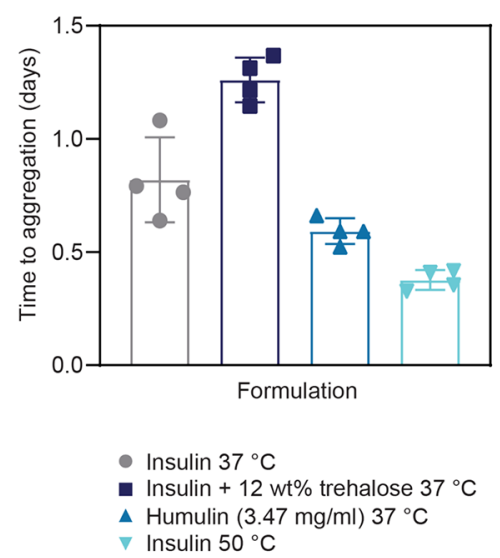

Figure 3. In vitro insulin stability as determined by the thioflavin $\mathrm{T}(\mathrm{ThT})$ insulin aggregation assay. All formulations were prepared at $10 \mathrm{mg} / \mathrm{mL}$ insulin unless otherwise indicated ( $n=4$ per formulation). 2:10 hydrogel formulations were loaded with $6.7 \mathrm{mg} / \mathrm{mL}$ insulin. (a) ThT assay signal was verified to be unaffected by the presence of the hydrogel components. (b, c) 1:X and 2:X hydrogel formulations are compared at stressed aging conditions of $37^{\circ} \mathrm{C}$ and constant agitation to simulate hot summer conditions. (d) Insulin formulated with a subset of hydrogel and polymer formulations aged at $50{ }^{\circ} \mathrm{C}$ with constant agitation to simulate extreme exposure conditions. (e) Stability comparison with the standard excipient trehalose and the commercial formulation Humulin. (f) Comparison of aggregation time for unencapsulated insulin formulation controls $(n=4$, mean \pm std. dev.).

as the shear rate approaches $100 \mathrm{~s}^{-1}$ for hydrogel formulations with NP content of 1 or 2 wt \%. (Figure $2 \mathrm{~b}$ ). The neat polymer solutions behaved as viscous liquids. At low shear $\left(0.01 \mathrm{~s}^{-1}\right)$, an approximately 3 orders of magnitude increase in viscosity was observed by increasing the NP content from 0 to 10 wt \% for 1:X and 2:X hydrogel formulations, respectively. A nearly 3 order of magnitude decrease in viscosity was observed for 1:5, $1: 10$, and 2:10 hydrogel formulations across the measured range of shear rates, despite their comparatively high storage moduli. This shear-thinning ability is attributed to the supramolecular, non-covalent nature of the interactions between the components of the hydrogel, allowing the network to break under high shear and reform under low shear conditions. In addition, the aforementioned rheological properties have been previously shown to translate to injectability through a syringe and needle or cathe- ter. $^{30,34,35,44,45}$ PNP hydrogels of various formulations have been shown to be both cytocompatible, as well as biocompatible in vivo in our previous work (Figure S11). ${ }^{34-36,44,45}$ Taken together, these results show a rheologically tunable hydrogel platform that meets the aforementioned criteria for a suitable material for insulin stabilization.

Thermal Stability of Hydrogel-Encapsulated Insulin. Storage of insulin under environmental conditions can lead to significant loss of drug activity for current commercial formulations. ${ }^{46}$ To test the capacity of PNP hydrogels to stabilize insulin, insulin-loaded hydrogels were exposed to stressed aging conditions. Insulin was encapsulated under mild conditions by simply mixing the drug into the bulk hydrogel material at a concentration of $10 \mathrm{mg} / \mathrm{mL}(2: 10$ hydrogel at 6.7 $\mathrm{mg} / \mathrm{mL}$ ), which is nearly 3 times the typical commercial 

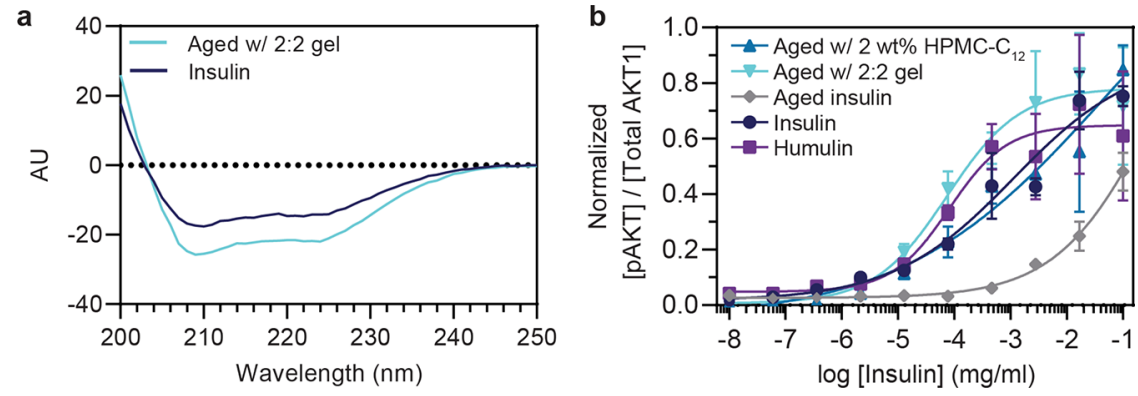

Figure 4. Secondary confirmation of insulin stability in PNP hydrogel. (a) Circular dichroism indicates the preservation of insulin secondary structure after 28 days of stressed aging $\left(37^{\circ} \mathrm{C}\right.$, constant agitation) within a 2:2 PNP hydrogel compared with a fresh insulin sample. (b) Functional activity of encapsulated insulin after 28 days of stressed aging $\left(37^{\circ} \mathrm{C}\right.$, constant agitation) was assessed via an in vitro cellular activity assay detecting insulin-stimulated phosphorylation of AKT and compared with the activity of fresh insulin and Humulin samples. Data shown are an average of $n=3$ aged sample replicates, mean \pm std. dev.

clinical formulation concentration of $100 \mathrm{U} / \mathrm{ml}(3.47 \mathrm{mg} / \mathrm{mL})$. The insulin-loaded samples were then subjected to stressed aging conditions $\left(37\right.$ or $50{ }^{\circ} \mathrm{C}$ with constant agitation) and the stability of the insulin was monitored via thioflavin $\mathrm{T}(\mathrm{ThT})$ assay (Figure S7). The primary mechanism of insulin denaturation is through the formation of amyloid fibrils, and ThT interaction with these fibrils results in a sharp increase in fluorescence signal indicating insulin aggregation. Individual gel components (polymer and nanoparticles) were tested as assay controls to confirm that they did not produce background fluorescence (Figures 3a and S8).

Samples were subjected to stressed aging conditions of 37 ${ }^{\circ} \mathrm{C}$ and constant agitation to simulate hot summer conditions, where room temperature storage may exceed $35^{\circ} \mathrm{C} .{ }^{46}$ Under these conditions, insulin formulated in phosphate buffered saline (PBS) aggregated in $20 \pm 4 \mathrm{~h}$ (Figure 3a). In contrast, when insulin was encapsulated in any PNP hydrogel formulation $(1: \mathrm{X}, 2: \mathrm{X})$ or formulated with neat polymer solutions ( 1 or 2 wt \% HPMC-C 12,2 wt \% HPMC), no aggregation was observed over the 28 day study (Figure $3 \mathrm{~b}, \mathrm{c}$ ). The termination of the study at 28 days was determined by the point where substantial evaporation of the formulations had taken place. Insulin formulated with only NPs $(2,5$, and $10 \mathrm{wt}$ $\%$ ) aggregated after less than $7 \mathrm{~h}$ on average (Figure S8). Together, these results suggest that the HPMC polymer contributes significantly to insulin stability in these systems.

To further test the capacity of selected top-performing formulations to withstand insulin aggregation under extreme thermal conditions, we conducted an additional stressed aging study at $50{ }^{\circ} \mathrm{C}$ with constant agitation. These conditions simulate an interruption in the cold chain where formulations are left unrefrigerated in a hot truck or shipping container. ${ }^{47}$ Insulin unfolds at approximately $70{ }^{\circ} \mathrm{C}$; therefore, we expect accelerated aggregation but not complete thermal denaturation at this temperature. ${ }^{48}$ Insulin in PBS aggregated in $9 \pm 1 \mathrm{~h}$, whereas all other formulations prevented aggregation for up to 12 days (Figure $3 \mathrm{~d}$ ). The termination of the study at 12 days was again determined by the point where substantial evaporation of the formulations had taken place. We anticipate that these systems may stabilize insulin well beyond the duration of these experiments when properly sealed in commercial manufacturing vials.

We also compared the stability of our formulations against two controls, trehalose and Humulin R (U100, $3.47 \mathrm{mg} / \mathrm{mL}$, Eli Lilly), under stressed aging conditions of $37{ }^{\circ} \mathrm{C}$ and constant agitation (Figure 3e,f). Trehalose is a commonly used stabilizing excipient for pharmaceuticals. ${ }^{9}$ While it is not used in current commercial insulin formulations, it serves as a benchmark to compare with the performance of other polymer and hydrogel systems for thermal stabilization reported in literature. Trehalose concentrations of 2, 6, and 12 wt \% were chosen to match the total solid polymer content in selected hydrogel formulations. Addition of trehalose to insulin formulated in PBS prevented aggregation for $30 \pm 2 \mathrm{~h}$ (12 wt $\%$ trehalose), indicating that the trehalose does indeed improve insulin stability to stressed aging; however, the observed aggregation times are significantly shortly than observed for the PNP hydrogels. Aggregation time is not reported for hydrogel and polymer formulations as they remained stable for the entire duration of the study. The commercial insulin formulation Humulin, which is formulated with zinc and other small molecule excipients, aggregated in 14 $\pm 1 \mathrm{~h}$.

ThT assay results were corroborated by assessing both the secondary structure and in vitro bioactivity of gel-encapsulated insulin post-aging. Selected samples taken from formulations after 28 days of stressed aging $\left(37^{\circ} \mathrm{C}\right.$, constant agitation) were analyzed by circular dichroism to characterize the secondary protein structure and by an in vitro cellular activity assay to assess insulin function. Circular dichroism shows that the secondary structure of gel-encapsulated insulin is intact and comparable to fresh insulin, as indicated by the presence of peaks near 208 and $224 \mathrm{~nm}$ that are characteristic of $\alpha$-helices, the dominant secondary structure in hexameric insulin (Figures $4 \mathrm{a}$ and S9).

To verify the activity of gel-encapsulated insulin samples, dose-response curves were determined by an AlphaLISA (Perkin-Elmer) assay to quantify the ratio of phosphorylated AKT compared to total AKT in $\mathrm{C} 2 \mathrm{C} 12$ cells stimulated with aged insulin samples compared to fresh insulin controls. Our results indicate that insulin formulated with 2 wt \% $\mathrm{HPMC}-\mathrm{C}_{12}$ and 2:2 hydrogel exhibited activity comparable to both freshly prepared insulin, as well as Humulin, whereas aged insulin formulated only in PBS exhibited significantly reduced activity (activity, $\log \mathrm{EC} 50=$ mean \pm s.e. $(n=3): 2$ wt \% HPMC-C $\mathrm{C}_{12}$ $\log \mathrm{EC} 50=-1.3 \pm 2,2: 2$ hydrogel $\log \mathrm{EC} 50=-4.1 \pm 0.2$, aged insulin $\log \mathrm{EC} 50=-0.48 \pm 1.3$, insulin $\log \mathrm{EC} 50=-2.9 \pm 0.4$, Humulin $\operatorname{logEC50}=-4.1 \pm 0.2$ ) (Figure 4b). The logEC50 of insulin formulated in a 2:2 hydrogel was lower (indicating higher activity) compared to that of aged insulin in PBS $(p<$ $0.0001)$. No difference was observed between insulin formulated in a 2:2 hydrogel and fresh Humulin ( $p=$ 
0.8984). The ability of the PNP hydrogels to preserve insulin secondary structure and in vitro activity demonstrates their potential to reduce the cold chain dependence for insulin distribution, and moving forward, to serve as a sustained release drug delivery depot imparting enhanced drug stability at increased concentrations under physiological conditions.

Determining the precise mechanism through which these materials stabilize insulin is beyond the scope of the present work; however, it is known that the air-liquid interface can mediate insulin fibrillation by allowing for partial protein unfolding as insulin adsorbs to the surface, which can lead to insulin aggregate nucleation. ${ }^{49,50}$ We hypothesize that these materials improve thermal stability of insulin by limiting access to the air-water interface due to their high viscosity compared to a buffer solution. In addition, hydrogels can slow the diffusion of drug molecules, which may also contribute to their ability to promote thermal stability of biotherapeutics. ${ }^{51-54}$

Release of Drug Cargo by Simple Dilution. A key design component for clinical translation of hydrogel-stabilized biotherapeutics is the ability to either directly administer the hydrogel formulation, or easily separate the drug from the hydrogel encapsulant. ${ }^{20}$ The supramolecular PNP hydrogels can be diluted to disrupt the non-covalent polymer network and injected without any further processing steps (Video S1), reducing the potential impact on the pharmacokinetics of the encapsulated drug cargo. The biocompatible hydrogel components do not need to be separated from the biotherapeutic prior to use. ${ }^{30,36}$

The viscosity and shear-thinning profiles of both the 2:10 hydrogel and 2:2 hydrogel were measured to demonstrate the change in rheological properties upon serial dilution with PBS (Figures 5 and S10). For the 2:10 hydrogel, which has the highest storage modulus of all of the materials evaluated, $4 \times$ dilution with PBS buffer is sufficient to cause approximately 2 orders of magnitude decrease in viscosity (Figure 5a,b). For the 2:2 hydrogel, a $2 \times$ dilution with PBS buffer decreases viscosity by an order of magnitude (Figure 5d,e and Video S1). An approximate gel-to-sol transition was determined by inverted vial test, indicating gel-to-sol transitions upon $4 \times$ and $2 \times$ dilution for the 2:10 and 2:2 hydrogel formulations, respectively (Figure 5c,f). Because these hydrogels enhance the stability of insulin, insulin could be formulated at higher concentrations and simply diluted to standard concentrations before administration. There is clinical precedent for dilution or reconstitution prior to use for lyophilized drugs and vaccines, highlighting the utility of this simple approach for treatment of diabetes in low-resource environments. Further, 1:0, 1:1, 1:2, and 2:0 formulations enhanced insulin stability and demonstrated fluid-like properties without dilution. Thus, insulin could be formulated in these materials at standard concentrations and dilution would be unnecessary. Future work could include determining a lower threshold of polymer concentration needed for effective thermal stabilization.

\section{CONCLUSION}

We demonstrate that self-assembled physical hydrogels composed of an HPMC- $\mathrm{C}_{12}$ network non-covalently crosslinked by biodegradable core-shell nanoparticles are capable of extending the thermal stability of insulin at increased concentrations, elevated temperatures, and under constant mechanical agitation. Our physical hydrogel is mildly formulated to encapsulate biotherapeutics, effectively stabilizes insulin, and can be easily diluted for injection such that drug
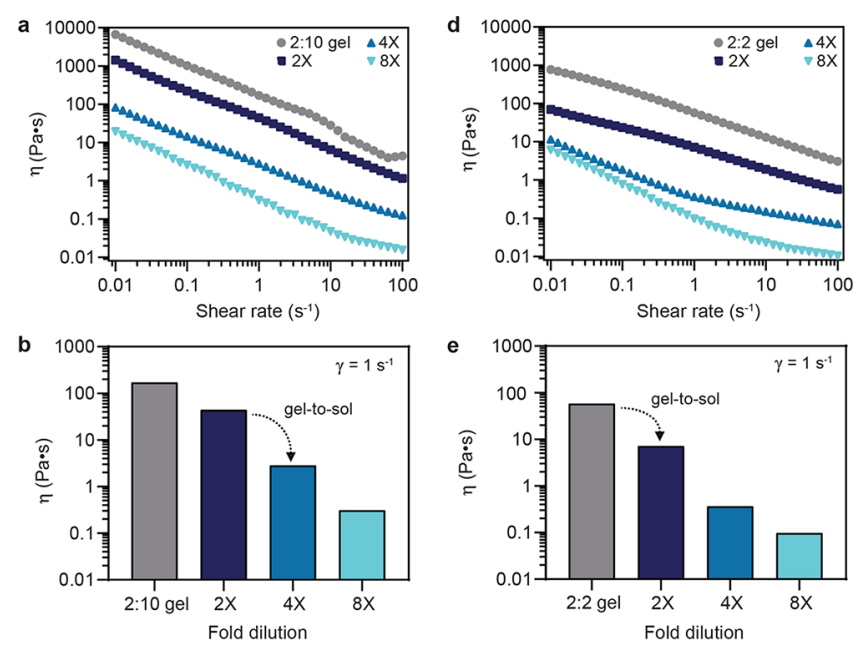

c
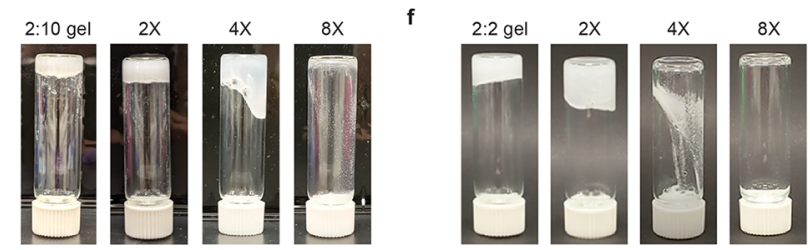

Figure 5. Supramolecular hydrogels can be diluted to disrupt the gel network and release drug cargo. (a, b) For the stiffest gel formulation (2:10), $4 \times$ dilution with PBS buffer allows the material to flow. Bar chart data at $1 \mathrm{~s}^{-1}$ shear rate. (c) Inverted vial test indicating an approximate gel-to-sol transition upon $4 \times$ dilution of the 2:10 gel formulation. (d, e) For the significantly less stiff 2:2 gel formulation, a $2 \times$ dilution with PBS buffer allows the material to flow. (f) Inverted vial test indicating an approximate gel-to-sol transition upon $2 \times$ dilution of the 2:2 gel formulation.

pharmacokinetics are unlikely to be affected. As the hydrogel components are both biocompatible and non-toxic, they would not need to be removed prior to administration. Because other biotherapeutics are also prone to denaturation and aggregation like insulin, this hydrogel platform would likely be able to provide thermal stability to other relevant research-grade and therapeutic macromolecules such as proteins and enzymes. We anticipate that the use of PNP hydrogels can help increase the global availability of insulin, and other biotherapeutics, in regions with unreliable cold chain infrastructure. Further, these hydrogels are being evaluated as injectable materials for sustained drug delivery, potentially enabling new formulation options and treatment strategies for insulin derivatives and macromolecular therapeutics more broadly as a drug class.

\section{ASSOCIATED CONTENT}

Supporting Information

The Supporting Information is available free of charge at https://pubs.acs.org/doi/10.1021/acsbiomaterials.0c01306.

Additional figures and methods (PDF)

Video S1: Injection and dilution of 2:2 PNP formulation (MOV)

\section{AUTHOR INFORMATION}

\section{Corresponding Author}

Eric A. Appel - Department of Materials Science \& Engineering, Department of Bioengineering, and ChEM-H Institute, Stanford University, Stanford, California 94305, United States; Department of Pediatrics-Endocrinology, 
Stanford University School of Medicine, Stanford, California 94305, United States; 이이.org/0000-0002-2301-7126; Email: eappel@stanford.edu

\section{Authors \\ Catherine M. Meis - Department of Materials Science \& Engineering, Stanford University, Stanford, California 94305, United States \\ Erika E. Salzman - Department of Materials Science \& Engineering, Stanford University, Stanford, California 94305, United States \\ Caitlin L. Maikawa - Department of Bioengineering, Stanford University, Stanford, California 94305, United States \\ Anton A. A. Smith - Department of Science and Technology, Aarhus University, 8000 Aarhus, Denmark; Department of Materials Science \& Engineering, Stanford University, Stanford, California 94305, United States \\ Joseph L. Mann - Department of Materials Science \& Engineering, Stanford University, Stanford, California 94305, United States \\ Abigail K. Grosskopf - Department of Chemical Engineering, Stanford University, Stanford, California 94305, United States}

Complete contact information is available at:

https://pubs.acs.org/10.1021/acsbiomaterials.0c01306

\section{Notes}

The authors declare no competing financial interest.

\section{ACKNOWLEDGMENTS}

This work was funded in part by an NIDDK R01 (NIH Grant R01DK119254), a Pilot \& Feasibility seed grant from the Stanford Diabetes Research Center (NIH Grant P30DK116074), the Center for Human Systems Immunology with Bill \& Melinda Gates Foundation (OPP1113682), and the Bill \& Melinda Gates Foundation (OPP1211043). C.M.M. was supported by a Stanford Graduate Fellowship and a Tau Beta Pi Graduate Fellowship. E.E.S. was supported by the Caltech Summer Undergraduate Research Fellowship program. C.L.M. was supported by the NSERC Postgraduate Scholarship and the Stanford BioX Bowes Graduate Student Fellowship. A.A.A.S. was funded by grant NNF18OC0030896 from the Novo Nordisk Foundation and the Stanford Bio-X Program, and also funded by the Danish Council of Independent Research (Grant No. DFF505400215). J.L.M. was supported by the Department of Defense NDSEG Fellowship and by a Stanford Graduate Fellowship. A.K.G. is thankful for a National Science Foundation Graduate Research Fellowship and the Gabilan Fellowship of the Stanford Graduate Fellowship in Science and Engineering. The authors acknowledge the High-Throughput Bioscience Center (HTBC) at Stanford Medicine. The authors acknowledge Anthony C. Yu for his assistance with hydrogel formulation protocols and supplemental video material. The authors acknowledge Dr. Jaime Cheah and Dr. Abel Cortinas for advice regarding the AlphaLISA assay protocol.

\section{REFERENCES}

(1) Urquhart, L. Top drugs and companies by sales in 2018. Nat. Rev. Drug Discovery 2019, 18, 245.

(2) Turner, M. R.; Balu-Iyer, S. V. Challenges and opportunities for the subcutaneous delivery of therapeutic proteins. J. Pharm. Sci. 2018, $107,1247-1260$.
(3) Mitragotri, S.; Burke, P. A.; Langer, R. Overcoming the challenges in administering biopharmaceuticals: formulation and delivery strategies. Nat. Rev. Drug Discovery 2014, 13, 655.

(4) Ashok, A.; Brison, M.; LeTallec, Y. Improving cold chain systems: Challenges and solutions. Vaccine 2017, 35, 2217-2223.

(5) Subzwari, M.; Nasir, S. Z. Preserving Efficacy of Temperature Sensitive Medicines-Logistics Management in Pharmaceutical Supply Chain. South Asian Journal of Management 2015, 9, 1-9.

(6) Sykes, C. Time-and Temperature-Controlled Transport: Supply Chain Challenges and Solutions. Pharmacy and Therapeutics 2018, 43, 154

(7) Kristensen, D. D.; Bartholomew, K.; Villadiego, S.; Lorenson, K. What vaccine product attributes do immunization program stakeholders value? Results from interviews in six low-and middle-income countries. Vaccine 2016, 34, 6236-6242.

(8) Basta, N.; Lipowicz, M. Biopharma Cold Chain Sourcebook, 10th ed.; 2019.

(9) Kamerzell, T. J.; Esfandiary, R.; Joshi, S. B.; Middaugh, C. R.; Volkin, D. B. Protein-excipient interactions: Mechanisms and biophysical characterization applied to protein formulation development. Adv. Drug Delivery Rev. 2011, 63, 1118-1159.

(10) Frokjaer, S.; Otzen, D. E. Protein drug stability: a formulation challenge. Nat. Rev. Drug Discovery 2005, 4, 298-306.

(11) Jain, D.; Mahammad, S. S.; Singh, P. P.; Kodipyaka, R. A review on parenteral delivery of peptides and proteins. Drug Dev. Ind. Pharm. 2019, 45, 1403-1420.

(12) Rayaprolu, B. M.; Strawser, J. J.; Anyarambhatla, G. Excipients in parenteral formulations: selection considerations and effective utilization with small molecules and biologics. Drug Dev. Ind. Pharm. 2018, 44, 1565-1571.

(13) Mann, J. L.; Maikawa, C. L.; Smith, A. A.; Grosskopf, A. K.; Baker, S. W.; Roth, G. A.; Meis, C. M.; Gale, E. C.; Liong, C. S.; Correa, S.; et al. An ultrafast insulin formulation enabled by highthroughput screening of engineered polymeric excipients. Sci. Transl. Med. 2020, 12, eaba6676.

(14) Wei, W.; Qi, X.; Li, J.; Zuo, G.; Sheng, W.; Zhang, J.; Dong, W. Smart macroporous salecan/poly (N, N-diethylacrylamide) semi-IPN hydrogel for anti-inflammatory drug delivery. ACS Biomater. Sci. Eng. 2016, 2, 1386-1394.

(15) Qi, X.; Wei, W.; Li, J.; Liu, Y.; Hu, X.; Zhang, J.; Bi, L.; Dong, W. Fabrication and characterization of a novel anticancer drug delivery system: salecan/poly (methacrylic acid) semi-interpenetrating polymer network hydrogel. ACS Biomater. Sci. Eng. 2015, 1, 1287-1299.

(16) Qi, X.; Wei, W.; Li, J.; Zuo, G.; Pan, X.; Su, T.; Zhang, J.; Dong, W. Salecan-based $\mathrm{pH}$-sensitive hydrogels for insulin delivery. Mol. Pharmaceutics 2017, 14, 431-440.

(17) Tibbitt, M. W.; Dahlman, J. E.; Langer, R. Emerging frontiers in drug delivery. J. Am. Chem. Soc. 2016, 138, 704-717.

(18) Li, J.; Mooney, D. J. Designing hydrogels for controlled drug delivery. Nature Reviews Materials 2016, 1, 16071.

(19) Fenton, O. S.; Tibbitt, M. W.; Appel, E. A.; Jhunjhunwala, S.; Webber, M. J.; Langer, R. Injectable Polymer-Nanoparticle Hydrogels for Local Immune Cell Recruitment. Biomacromolecules 2019, 20, $4430-4436$

(20) Welch, R. P.; Lee, H.; Luzuriaga, M. A.; Brohlin, O. R.; Gassensmith, J. J. Protein-Polymer Delivery: Chemistry from the Cold Chain to the Clinic. Bioconjugate Chem. 2018, 29, 2867-2883.

(21) Sawada, S.; Akiyoshi, K. Nano-encapsulation of lipase by selfassembled nanogels: induction of high enzyme activity and thermal stabilization. Macromol. Biosci. 2010, 10, 353-358.

(22) Pritchard, E. M.; Dennis, P. B.; Omenetto, F.; Naik, R. R.; Kaplan, D. L. Physical and chemical aspects of stabilization of compounds in silk. Biopolymers 2012, 97, 479-498.

(23) Akiyoshi, K.; Kobayashi, S.; Shichibe, S.; Mix, D.; Baudys, M.; Wan Kim, S.; Sunamoto, J. Self-assembled hydrogel nanoparticle of cholesterol-bearing pullulan as a carrier of protein drugs: complexation and stabilization of insulin. J. Controlled Release 1998, 54, 313320. 
(24) Zhang, P.; Sun, F.; Tsao, C.; Liu, S.; Jain, P.; Sinclair, A.; Hung, H.-C.; Bai, T.; Wu, K.; Jiang, S. Zwitterionic gel encapsulation promotes protein stability, enhances pharmacokinetics, and reduces immunogenicity. Proc. Natl. Acad. Sci. U. S. A. 2015, 112, 1204612051.

(25) Mann, J. L.; Yu, A. C.; Agmon, G.; Appel, E. A. Supramolecular polymeric biomaterials. Biomater. Sci. 2018, 6, 10-37.

(26) Sridhar, B. V.; Janczy, J. R.; Hatlevik, Ø.; Wolfson, G.; Anseth, K. S.; Tibbitt, M. W. Thermal stabilization of biologics with photoresponsive hydrogels. Biomacromolecules 2018, 19, 740-747.

(27) Lee, J.; Ko, J. H.; Lin, E.-W.; Wallace, P.; Ruch, F.; Maynard, H. D. Trehalose hydrogels for stabilization of enzymes to heat. Polym. Chem. 2015, 6, 3443-3448.

(28) Lee, J.; Ko, J. H.; Mansfield, K. M.; Nauka, P. C.; Bat, E.; Maynard, H. D. Glucose-Responsive Trehalose Hydrogel for Insulin Stabilization and Delivery. Macromol. Biosci. 2018, 18, 1700372.

(29) Panescu, P. H.; Ko, J. H.; Maynard, H. D. Scalable TrehaloseFunctionalized Hydrogel Synthesis for High-Temperature Protection of Enzymes. Macromol. Mater. Eng. 2019, 304, 1800782.

(30) Appel, E. A.; Tibbitt, M. W.; Webber, M. J.; Mattix, B. A.; Veiseh, O.; Langer, R. Self-assembled hydrogels utilizing polymernanoparticle interactions. Nat. Commun. 2015, 6, 6295.

(31) Yu, A. C.; Smith, A. A. A.; Appel, E. A. Structural considerations for physical hydrogels based on polymer-nanoparticle interactions. Molecular Systems Design \& Engineering 2020, 5, 401407.

(32) Yu, A. C.; Lopez Hernandez, H.; Kim, A. H.; Stapleton, L. M.; Brand, R. J.; Mellor, E. T.; Bauer, C. P.; McCurdy, G. D.; Wolff, A. J.; Chan, D.; Criddle, C. S.; Acosta, J. D.; Appel, E. A. Wildfire prevention through prophylactic treatment of high-risk landscapes using viscoelastic retardant fluids. Proc. Natl. Acad. Sci. U. S. A. 2019, 116, 20820-20827.

(33) Yu, A. C.; Chen, H.; Chan, D.; Agmon, G.; Stapleton, L. M.; Sevit, A. M.; Tibbitt, M. W.; Acosta, J. D.; Zhang, T.; Franzia, P. W.; Langer, R.; Appel, E. A. Scalable manufacturing of biomimetic moldable hydrogels for industrial applications. Proc. Natl. Acad. Sci. U. S. A. 2016, 113, 14255-14260.

(34) Grosskopf, A. K.; Roth, G. A.; Smith, A. A.; Gale, E. C.; Hernandez, H. L.; Appel, E. A. Injectable supramolecular polymernanoparticle hydrogels enhance human mesenchymal stem cell delivery. Bioengineering \& Translational Medicine 2020, 5, e10147.

(35) Lopez Hernandez, H.; Grosskopf, A. K.; Stapleton, L. M.; Agmon, G.; Appel, E. A. Non-Newtonian polymer-nanoparticle hydrogels enhance cell viability during injection. Macromol. Biosci. 2019, 19, 1800275.

(36) Stapleton, L. M.; Steele, A. N.; Wang, H.; Lopez Hernandez, H.; Yu, A. C.; Paulsen, M. J.; Smith, A. A. A.; Roth, G. A.; Thakore, A. D.; Lucian, H. J.; Totherow, K. P.; Baker, S. W.; Tada, Y.; Farry, J. M.; Eskandari, A.; Hironaka, C. E.; Jaatinen, K. J.; Williams, K. M.; Bergamasco, H.; Marschel, C.; Chadwick, B.; Grady, F.; Ma, M.; Appel, E. A.; Woo, Y. J. Use of a supramolecular polymeric hydrogel as an effective post-operative pericardial adhesion barrier. Nature Biomedical Engineering 2019, 3, 611-620.

(37) Roth, G. A.; Gale, E. C.; Alcántara-Hernández, M.; Luo, W.; Axpe, E.; Verma, R.; Yin, Q.; Yu, A. C.; Lopez Hernandez, H.; Maikawa, C. L.; Smith, A. A. A.; Davis, M. M.; Pulendran, B.; Idoyaga, J.; Appel, E. A. Injectable hydrogels for sustained co-delivery of subunit vaccines enhance humoral immunity. ACS Cent. Sci. 2020, 6, 1800

(38) Garg, S. K.; Rewers, A. H.; Akturk, H. K. Ever-increasing insulin-requiring patients globally. Diabetes Technol. Ther. 2018, 20, S2-1-S2-4.

(39) Webber, M. J.; Appel, E. A.; Vinciguerra, B.; Cortinas, A. B.; Thapa, L. S.; Jhunjhunwala, S.; Isaacs, L.; Langer, R.; Anderson, D. G. Supramolecular PEGylation of biopharmaceuticals. Proc. Natl. Acad. Sci. U. S. A. 2016, 113, 14189-14194.

(40) Maikawa, C. L.; Smith, A. A.; Zou, L.; Meis, C. M.; Mann, J. L.; Webber, M. J.; Appel, E. A. Stable Monomeric Insulin Formulations
Enabled by Supramolecular PEGylation of Insulin Analogues. Advanced Therapeutics 2020, 3, 1900094.

(41) Maikawa, C. L.; Smith, A. A. A.; Zou, L.; Roth, G. A.; Gale, E. C.; Stapleton, L. M.; Baker, S. W.; Mann, J. L.; Yu, A. C.; Correa, S.; Grosskopf, A. K.; Liong, C. S.; Meis, C. M.; Chan, D.; Troxell, M.; Maahs, D. M.; Buckingham, B. A.; Webber, M. J.; Appel, E. A. A coformulation of supramolecularly stabilized insulin and pramlintide enhances mealtime glucagon suppression in diabetic pigs. Nature Biomedical Engineering 2020, 4, 507-517.

(42) IDF Diabetes Atlas, 9th ed., 2019. https://www.diabetesatlas. org.

(43) Schlein, M. Insulin Formulation Characterization-The Thioflavin T Assays. AAPS J. 2017, 19, 397-408.

(44) Steele, A. N.; Stapleton, L. M.; Farry, J. M.; Lucian, H. J.; Paulsen, M. J.; Eskandari, A.; Hironaka, C. E.; Thakore, A. D.; Wang, H.; Yu, A. C.; Chan, D.; Appel, E. A.; Woo, Y. J. A Biocompatible Therapeutic Catheter-Deliverable Hydrogel for In Situ Tissue Engineering. Adv. Healthcare Mater. 2019, 8, 1801147.

(45) Steele, A. N.; Paulsen, M. J.; Wang, H.; Stapleton, L. M.; Lucian, H. J.; Eskandari, A.; Hironaka, C. E.; Farry, J. M.; Baker, S. W.; Thakore, A. D.; Jaatinen, K. J.; Tada, Y.; Hollander, M. J.; Williams, K. M.; Seymour, A. J.; Totherow, K. P.; Yu, A. C.; Cochran, J. R.; Appel, E. A.; Woo, Y. J. Multi-phase catheter-injectable hydrogel enables dual-stage protein-engineered cytokine release to mitigate adverse left ventricular remodeling following myocardial infarction in a small animal model and a large animal model. Cytokine+ 2020, 127, 154974.

(46) Khurana, G.; Gupta, V. Effect on Insulin upon Storage in Extreme Climatic Conditions (Temperature and Pressure) and Their Preventive Measures. Journal of Social Health and Diabetes 2019, 7, 610.

(47) Schafer, H. Field-Measured Temperature Profiles of Truck-Carried Materiel in Desert, Tropic, Mountainous Temperate, and Arctic Locations; Naval Weapons Center, 1983; pp 1-75.

(48) Huus, K.; Havelund, S.; Olsen, H. B.; van de Weert, M.; Frokjaer, S. Thermal dissociation and unfolding of insulin. Biochemistry 2005, 44, 11171-11177.

(49) Nielsen, L.; Khurana, R.; Coats, A.; Frokjaer, S.; Brange, J.; Vyas, S.; Uversky, V. N.; Fink, A. L. Effect of Environmental Factors on the Kinetics of Insulin Fibril Formation: Elucidation of the Molecular Mechanism. Biochemistry 2001, 40, 6036-6046.

(50) Sluzky, V.; Klibanov, A. M.; Langer, R. Mechanism of insulin aggregation and stabilization in agitated aqueous solutions. Biotechnol. Bioeng. 1992, 40, 895-903.

(51) Axpe, E.; Chan, D.; Offeddu, G. S.; Chang, Y.; Merida, D.; Hernandez, H. L.; Appel, E. A. A multiscale model for solute diffusion in hydrogels. Macromolecules 2019, 52, 6889-6897.

(52) Ritger, P. L.; Peppas, N. A. A simple equation for description of solute release I. Fickian and non-fickian release from non-swellable devices in the form of slabs, spheres, cylinders or discs. J. Controlled Release 1987, 5, 23-36.

(53) Reinhart, C. T.; Peppas, N. A. Solute diffusion in swollen membranes. Part II. Influence of crosslinking on diffusive properties. J. Membr. Sci. 1984, 18, 227-239.

(54) Peppas, N.; Bures, P.; Leobandung, W.; Ichikawa, H. Hydrogels in pharmaceutical formulations. Eur. J. Pharm. Biopharm. 2000, 50, $27-46$. 